\title{
SOME APPLICATIONS OF SEMI-DISCRETE VARIATIONAL INTEGRATORS TO CLASSICAL FIELD THEORIES
}

\author{
M. DE LEÓN, JUAN C. MARRERO, AND DAVID MARTÍN DE DIEGO
}

\begin{abstract}
We develop a semi-discrete version of discrete variational mechanics with applications to numerical integration of classical field theories. The geometric preservation properties are studied.
\end{abstract}

\section{INTRODUCTION}

The calculus of variations is a fundamental tool in the description and understanding of Classical Mechanics and it is an area of active research. One part of this activity was dedicated to uncover the geometrical structures behind such formalisms. Many physical systems not only evolve in time, as in Classical Mechanics, but also posses a continuous spatial structure. This is the setting of Classical Field Theories in both the Lagrangian and Hamiltonian formalisms [2, 3].

One traditional way to analyze these problems has been to pass to the Hamiltonian formalism (or Lagrangian formalism) using a space-time decomposition of the parameter space, and then applying classical Dirac's theory of constraints (in the singular case, which is typical in field theories). In this way, we obtain a well-posed system of equations of motion that can be eventually integrated or numerically simulated. It is clear that using this space-time decomposition we broke the original covariance of the theory and perhaps some geometrical structure is lost, but the treatment of the equations is, in many aspects, more easy and some of the geometrical structure is still preserved. Moreover, for numerical simulation of the equations of motion, after the space-time decomposition, we eventually obtain a Hamiltonian system and symplectic integration methods may be useful to solve numerically the initial problem.

In this sense, it may be useful to introduce geometric integrators, that is, numerical schemes which preserve some of the extra features of geometric nature of the dynamical systems. Usually, these integrators can run, in simulations, for long time with lower spurious effects (for instance, bad energy behavior for conservative systems) than the traditional ones [6, 17.

A particular case of geometric integrators are variational integrators. These integrators have their roots in the optimal control literature in the 1960's and they enter in the "geometric differential arena" after the pioneering work of Veselov [19] and Moser and Veselov [15, 19]. In these papers, there appears the discrete action sum, discrete Euler-Lagrange equations, discrete Noether theorem... These integrators have been adapted for Field Theories [7, 10. All these integrators have demonstrated exceptionally good longtime behavior.

In this paper, we will develop the theory of semi-discrete variational integrators for Classical Field Theories. The basic idea is to consider an spatial truncation that reduces the partial differential equations derived from the Euler-Lagrange equations to a system of ordinary differential equations [8, 16. The main objective of the paper is to study the geometric properties after this spatial truncation: preservation of forms, energy preservation and momentum preservation.

Key words and phrases. Discrete mechanics, Classical Field theories, nonlinear wave equation, Lagrangian Mechanics, Hamiltonian Mechanics.

This work has been partially supported by MICYT (Spain) Grants BMF 2003-01319, MTM 2004-7832 and S-0505/ESP/0158 of the CAM. 


\section{Discrete variational Calculus}

First, we will recall discrete variational calculus, following the approach in [12 and references therein. A discrete Lagrangian is a map $L_{d}: Q \times Q \rightarrow \mathbb{R}$, which may be considered as an approximation of a continuous Lagrangian $L: T Q \rightarrow \mathbb{R}$. Define the action sum $S_{d}: Q^{N+1} \rightarrow \mathbb{R}$ corresponding to the Lagrangian $L_{d}$ by

$$
S_{d}\left(q_{0}, \ldots, q_{N}\right)=\sum_{k=1}^{N} L_{d}\left(q_{k-1}, q_{k}\right),
$$

where $q_{k} \in Q$ for $0 \leq k \leq N$.

Observe that for any covector $\alpha \in T_{\left(x_{1}, x_{2}\right)}^{*}(Q \times Q)$, we have the decomposition $\alpha=\alpha_{1}+\alpha_{2}$ where $\alpha_{i} \in T_{x_{i}}^{*} Q$, thus,

$$
d L_{d}\left(q_{0}, q_{1}\right)=D_{1} L_{d}\left(q_{0}, q_{1}\right)+D_{2} L_{d}\left(q_{0}, q_{1}\right) .
$$

The discrete variational principle states that the solutions of the discrete system determined by $L_{d}$ must extremize the action sum given fixed points $q_{0}$ and $q_{N}$. Extremizing $S_{d}$ over $q_{k}$, $1 \leq k \leq N-1$, we obtain the following system of difference equations

$$
D_{1} L_{d}\left(q_{k}, q_{k+1}\right)+D_{2} L_{d}\left(q_{k-1}, q_{k}\right)=0 .
$$

These equations are usually called the discrete Euler-Lagrange equations. Under some regularity hypothesis (the matrix $\left(D_{12} L_{d}\left(q_{k}, q_{k+1}\right)\right)$ is regular), it is possible to define a (local) discrete flow $\Upsilon: Q \times Q \longrightarrow Q \times Q$, by $\Upsilon\left(q_{k-1}, q_{k}\right)=\left(q_{k}, q_{k+1}\right)$ from Equations (2.1).

Define the discrete Legendre transformations associated to $L_{d}$ by

$$
\begin{aligned}
& F L_{d}^{-}: \quad Q \times Q \longrightarrow T^{*} Q \\
& \left(q_{0}, q_{1}\right) \longmapsto\left(q_{0},-D_{1} L_{d}\left(q_{0}, q_{1}\right)\right), \\
& F L_{d}^{+}: Q \times Q \quad \longrightarrow T^{*} Q \\
& \left(q_{0}, q_{1}\right) \longmapsto\left(q_{1}, D_{2} L_{d}\left(q_{0}, q_{1}\right)\right),
\end{aligned}
$$

and the 2 -form $\omega_{d}=\left(F L_{d}^{+}\right)^{*} \omega_{Q}=\left(F L_{d}^{-}\right)^{*} \omega_{Q}$, where $\omega_{Q}$ is the canonical symplectic form on $T^{*} Q$. The discrete algorithm determined by $\Upsilon$ preserves the symplectic form $\omega_{d}$, i.e., $\Upsilon^{*} \omega_{d}=\omega_{d}$. Moreover, if the discrete Lagrangian is invariant under the diagonal action of a Lie group $G$, then the discrete momentum map $J_{d}: Q \times Q \rightarrow \mathfrak{g}^{*}$ defined by $\left\langle J_{d}\left(q_{k}, q_{k+1}\right), \xi\right\rangle=\left\langle D_{2} L_{d}\left(q_{k}, q_{k+1}\right), \xi_{Q}\left(q_{k+1}\right)\right\rangle$ is preserved by the discrete flow. Therefore, these integrators are symplectic-momentum preserving integrators. Here, $\xi_{Q}$ denotes the fundamental vector field determined by $\xi \in \mathfrak{g}$, where $\mathfrak{g}$ the Lie algebra of $G$.

\section{Variational Calculus in Classical Field Theories}

Consider a locally trivial fibration $\pi: Y \longrightarrow X$, where $Y$ is an $(m+n+1)$-dimensional manifold and $X$ is the parameter space usually equipped with a global decomposition in space and time, that is, $X=\mathbb{R} \times P, \operatorname{dim} P=n$. We shall also fix a volume form on $X$, that will be denoted by $\eta$. We can choose fibred coordinates $\left(x^{\mu}, y^{i}\right)$ in $Y$, so that $\pi\left(x^{\mu}, y^{i}\right)=\left(x^{\mu}\right)$, where $\left(x^{\mu}\right)=\left(x^{0}, x^{1}, \ldots, x^{n}\right)$ and $x^{0} \equiv t$ represents the time evolution. Assume that the volume form is $\eta=d x^{0} \wedge \ldots \wedge d x^{n}$. Here, $0 \leq \mu, \nu, \ldots \leq n$ and $1 \leq i, j, \ldots \leq m$. We shall also use the following useful notation $d^{n} x^{\mu}:=\iota_{\partial / \partial x^{\mu} \eta}$.

The first order jet prolongation $J^{1} \pi$ is the manifold of classes $j_{x}^{1} \phi$ of sections $\phi$ of $\pi$ (whose set will be denoted by $\Gamma(\pi)$ ) around a point $x$ of $X$ which have the same Taylor expansion up to order one. $J^{1} \pi$ can be viewed as the generalization of the phase space of the velocities for Classical Mechanics. Therefore, $J^{1} \pi$, which we shall denote by $Z$, is an $(n+1+m+(n+1) m)$-dimensional manifold. If we have adapted coordinates $\left(x^{\mu}, y^{i}\right)$ in $Y$, then we have induced coordinates in $Z$, given by

$$
\left(x^{\mu}, y^{i}, z_{\mu}^{i}\right)=\left(t, x^{1}, \ldots, x^{n}, y^{i}, z_{t}^{i}, z_{1}^{i}, \ldots, z_{n}^{i}\right) .
$$


Suppose that we are given a function $L: Z \longrightarrow \mathbb{R}$ of class $C^{2}$ in its $(n+1+m+(n+1) m)$ arguments. Let $G_{X}$ be a compact $(n+1)$-dimensional submanifold on $X$. We can thus construct the following functional

$$
\mathcal{J}_{L}(\phi)=\int_{G_{X}} L\left(x^{\mu}, y^{i}(x), y_{\mu}^{i}(x)\right) d x^{0} \wedge \ldots \wedge d x^{n}
$$

for all section $\phi \in \Gamma(\pi)$, where $\phi(x)=\left(x^{\mu}, y^{i}(x), y_{\mu}^{i}(x)\right)$

Definition 3.1. A section $\phi \in \Gamma(\pi)$ is a solution of the variational problem determined by $L$ if and only if $\phi$ is a critical point of $\mathcal{J}_{L}$.

Extremizing the functional $\partial_{L}$ we obtain the Euler-lagrange equations:

$$
\frac{\partial L}{\partial y^{i}}-\frac{d}{d x^{\mu}}\left(\frac{\partial L}{\partial z_{\mu}^{i}}\right)=0, \quad 0 \leq i \leq m
$$

Now, denote by $\hat{p}_{i}^{\mu}:=\frac{\partial L}{\partial z_{\mu}^{i}}$ and by $\hat{p}:=L-z_{\mu}^{i} \hat{p}_{i}^{\mu}$, then for a given Lagrangian function $L$ and a volume form $\eta$ we can construct the Poincaré-Cartan $(n+1)$-form

$$
\begin{aligned}
\Theta_{L} & =\left(L-z_{\mu}^{i} \frac{\partial L}{\partial z_{\mu}^{i}}\right) d^{n+1} x+\frac{\partial L}{\partial z_{\mu}^{i}} d y^{i} \wedge d^{n} x^{\mu} \\
& =\left(\hat{p} d x^{\mu}+\hat{p}_{i}^{\mu} d y^{i}\right) \wedge d^{n} x^{\mu}
\end{aligned}
$$

From this form, we can also define the Poincaré-Cartan $(n+2)$-form as $\Omega_{L}:=-d \Theta_{L}$. In induced coordinates is expressed as follows

$$
\Omega_{L}=-\left(d \hat{p} \wedge d x^{\mu}+d \hat{p}_{i}^{\mu} \wedge d y^{i}\right) \wedge d^{n} x^{\mu}
$$

¿From these equations it is easy to derive an intrinsic version of Euler-Lagrange equations. In fact, a section $\phi \in \Gamma(\pi)$ is an extremal of $\mathcal{\partial}_{L}$ if and only if

$$
\left(j^{1} \phi\right)^{*}\left(\iota_{\xi} \Omega_{L}\right)=0
$$

for every vector field $\xi$ on $Z$.

We refer the reader to reference 2] for more details and a complete derivation of the equations and the geometric framework for classical field theories.

\section{Motivating Example: The nonlinear waVe EQUation}

Consider the nonlinear wave equation given by

$$
u_{t t}=\delta_{x} \sigma^{\prime}\left(u_{x}\right)-f^{\prime}(u)
$$

where $u: U \subset \mathbb{R}^{2} \rightarrow \mathbb{R}$ and $\sigma, f$ are smooth functions. If $\sigma\left(u_{x}\right)=u_{x}^{2} / 2$ then we obtain the semi-linear wave equation.

Equation (4.1) corresponds to the Euler-Lagrange equation for the lagrangian function

$$
L\left(u, u_{t}, u_{x}\right)=\frac{1}{2} u_{t}^{2}-\sigma\left(u_{x}\right)-f(u)
$$

Observe that, in this particular case, the Lagrangian does not depend on the parameter space $X=\mathbb{R} \times \mathbb{R}$.

Now, replace the $x$-derivative in the Lagrangian by a simple difference (for simplicity, we will work with a uniform grid of $N+1$ points, $h=L / N)$ as follows:

$$
L_{s d}\left(u_{0}, u_{1},\left(u_{0}\right)_{t},\left(u_{1}\right)_{t}\right)=\frac{1}{2}\left(\frac{\left(u_{0}\right)_{t}+\left(u_{1}\right)_{t}}{2}\right)^{2}-\sigma\left(\frac{u_{1}-u_{0}}{h}\right)-f\left(\frac{u_{1}+u_{0}}{2}\right)
$$

In this case, the Lagrangian is a function $L_{s d}: T \mathbb{R} \times T \mathbb{R} \simeq T(\mathbb{R} \times \mathbb{R}) \longrightarrow \mathbb{R}$. This spatialdiscretization is useful, since as we will show in the next section, applying a suitable variational principle we obtain a semi-discretization of Equation (4.1), replacing the partial differential equation by a system of ordinary differential equations. After this step, the equations of motion can 
be integrated in time or numerically integrated using, for instance, a symplectic method as the symplectic Euler scheme.

In the next sections, we will show that with these semi-discretizations some of the geometric properties are preserved.

\section{Semi-Discrete Variational Calculus}

Given a smooth manifold $Q$ consider the following sets

$$
\begin{aligned}
\mathcal{C}_{N,[0, T]}= & \left\{y:\{0, \ldots, N\} \times[0, T] \longrightarrow Q \mid y(k, \cdot) \in C^{2}([0, T]) \text { for all } k\right. \\
& \text { and the curves } t \rightarrow y(0, t) \text { and } t \rightarrow y(N, t) \text { are fixed }\}, \\
\widetilde{\mathcal{C}}_{N,[0, T]=}\left\{y:\{0, \ldots, N\} \times[0, T] \longrightarrow Q \mid y(k, \cdot) \in C^{2}([0, T])\right. & \\
& \text { and the values } y(k, 0) \text { and } y(k, T) \text { are fixed for all } k\} .
\end{aligned}
$$

The choice of one of these sets depends of the different boundary conditions of the initial problem. Of course, another boundary conditions can be analyzed considering suitable adaptations of both situations.

Now, let $L: T Q \times T Q \simeq T(Q \times Q) \rightarrow \mathbb{R}$ be a Lagrangian function. If $\left(v_{0}, v_{1}\right) \in T_{q_{0}} Q \times T_{q_{1}} Q$ then, as in Section 2, we have that

$$
d L\left(v_{0}, v_{1}\right)=\left(\tilde{D}_{1} L\right)\left(v_{0}, v_{1}\right)+\left(\tilde{D}_{2} L\right)\left(v_{0}, v_{1}\right),
$$

with $\left(\tilde{D}_{1} L\right)\left(v_{0}, v_{1}\right) \in T_{v_{0}}^{*}(T Q)$ and $\left(\tilde{D}_{2} L\right)\left(v_{0}, v_{1}\right) \in T_{v_{1}}^{*}(T Q)$.

On the other hand, if $\left(q_{0}^{i}\right)$ (respectively, $\left.\left(q_{1}^{i}\right)\right)$ are local coordinates on an open subset $U_{0}$ (respectively, $\left.\left(U_{1}\right)\right)$ of $Q$ such that $q_{0} \in U_{0}$ (respectively, $q_{1} \in U_{1}$ ), then we may consider the corresponding local coordinates $\left(q_{0}^{i}, \dot{q}_{0}^{i}, q_{1}^{i}, \dot{q}_{1}^{i}\right)$ on $T Q \times T Q$ and it follows that

$$
\begin{aligned}
& \tilde{D}_{1} L\left(v_{0}, v_{1}\right)=\sum_{i=1}^{n}\left(\frac{\partial L}{\partial q_{0 \mid\left(v_{0}, v_{1}\right)}^{i}} d q_{0}^{i}\left(v_{0}, v_{1}\right)+\frac{\partial L}{\partial \dot{q}_{0 \mid\left(v_{0}, v_{1}\right)}^{i}} d \dot{q}_{0}^{i}\left(v_{0}, v_{1}\right)\right), \\
& \tilde{D}_{2} L\left(v_{0}, v_{1}\right)=\sum_{i=1}^{n}\left(\frac{\partial L}{\partial q_{1 \mid\left(v_{0}, v_{1}\right)}^{i}} d q_{1}^{i}\left(v_{0}, v_{1}\right)+\frac{\partial L}{\partial \dot{q}_{1 \mid\left(v_{0}, v_{1}\right)}^{i}} d \dot{q}_{1}^{i}\left(v_{0}, v_{1}\right)\right) \text {. }
\end{aligned}
$$

Moreover, we will use the following notation

$$
\begin{aligned}
& D_{1} L\left(v_{0}, v_{1}\right)=\sum_{i=1}^{n} \frac{\partial L}{\partial q_{0}^{i}} \underset{\left(v_{0}, v_{1}\right)}{n} d q_{0}^{i}\left(v_{0}, v_{1}\right), \\
& D_{2} L\left(v_{0}, v_{1}\right)=\sum_{i=1}^{n} \frac{\partial L}{\partial \dot{q}_{0 \mid\left(v_{0}, v_{1}\right)}^{i}} d \dot{q}_{0}^{i}\left(v_{0}, v_{1}\right), \\
& \left.D_{3} L\left(v_{0}, v_{1}\right)=\sum_{i=1}^{n} \frac{\partial L}{\partial q_{1}^{i}} d v_{0}, v_{1}\right) \\
& \left.D_{4} L\left(v_{0}, v_{1}\right)=\sum_{i=1}^{n} \frac{\partial L}{\partial \dot{q}_{1}^{i}} d v_{0}, v_{1}\right), \\
&
\end{aligned}
$$

Note that $D_{1} L\left(v_{0}, v_{1}\right)$ and $D_{3} L\left(v_{0}, v_{1}\right)$ may be considered as 1 -forms on $Q$ at the points $q_{0}$ and $q_{1}$, respectively. In fact,

$$
\begin{aligned}
& \left(\left(\tau_{Q}\right)_{*}^{v_{0}}\right)^{t}\left(\sum_{i=1}^{n} \frac{\partial L}{\partial q_{0 \mid\left(v_{0}, v_{1}\right)}^{i}} d q_{0}^{i}\left(q_{0}\right)\right)=D_{1} L\left(v_{0}, v_{1}\right), \\
& \left(\left(\tau_{Q}\right)_{*}^{v_{1}}\right)^{t}\left(\sum_{i=1}^{n} \frac{\partial L}{\partial q_{1 \mid\left(v_{0}, v_{1}\right)}^{i}} d q_{1}^{i}\left(q_{1}\right)\right)=D_{3} L\left(v_{0}, v_{1}\right),
\end{aligned}
$$

where $\left(\left(\tau_{Q}\right)_{*}^{v_{0}}\right)^{t}: T_{q_{0}}^{*} Q \rightarrow T_{v_{0}}^{*}(T Q)$ (respectively, $\left.\left(\left(\tau_{Q}\right)_{*}^{v_{1}}\right)^{t}: T_{q_{1}}^{*} Q \rightarrow T_{v_{1}}^{*}(T Q)\right)$ is the dual map of the linear epimorphism $\left(\tau_{Q}\right)_{*}^{v_{0}}: T_{v_{0}}(T Q) \rightarrow T_{q_{0}} Q$ (respectively, $\left.\left(\tau_{Q}\right)_{*}^{v_{1}}: T_{v_{1}}(T Q) \rightarrow T_{q_{1}} Q\right)$ ). In addition, using the canonical identification between the vector spaces $T_{q_{0}} Q$ and $\operatorname{ker}\left(\tau_{Q}\right)_{*}^{v_{0}}=<$ 
$\frac{\partial}{\partial \dot{q}_{0 \mid v_{0}}^{i}}>$ (respectively, $T_{q_{1}} Q$ and $\operatorname{ker}\left(\tau_{Q}\right)_{*}^{v_{1}}=<\frac{\partial}{\partial \dot{q}_{1 \mid v_{1}}^{i}}>$ ) we have that the 1-form $D_{2} L\left(v_{0}, v_{1}\right)$ (respectively, $\left.D_{4} L\left(v_{0}, v_{1}\right)\right)$ may be considered as the 1 -form on $Q$ at the point $q_{0}$ (respectively, $\left.q_{1}\right)$

$$
\sum_{i=1}^{n} \frac{\partial L}{\partial \dot{q}_{0 \mid\left(v_{0}, v_{1}\right)}^{i}} d q_{0}^{i}\left(q_{0}\right)
$$

(respectively, $\left.\sum_{i=1}^{n} \frac{\partial L}{\partial \dot{q}_{1 \mid\left(v_{0}, v_{1}\right)}^{i}} d q_{1}^{i}\left(q_{1}\right)\right)$.

5.1. Variational calculus on $\mathcal{C}_{N,[0, T]}$. Define the semi-discrete action $\mathcal{S}_{\mathrm{Sd}} L: \mathcal{C}_{N,[0, T]} \longrightarrow \mathbb{R}$ as follows

$$
\begin{aligned}
\mathcal{S}_{\mathrm{Sd}} L(y(\cdot, \cdot)) & =\int_{0}^{T}\left[\sum_{k=0}^{N-1} L(\dot{y}(k, t), \dot{y}(k+1, t))\right] d t \\
& =\int_{0}^{T}\left[\sum_{k=0}^{N-1} L(y(k, t), \dot{y}(k, t), y(k+1, t), \dot{y}(k+1, t))\right] d t .
\end{aligned}
$$

Definition 5.1. An element $y \in \mathcal{C}_{N,[0, T]}$ is a solution of the semi-discrete variational problem determined by $L$ if and only if it is a critical point of the Lagrangian system defined by $\mathcal{S}_{s d} L$.

Therefore, a solution $y$ of the semi-discrete variational problem extremizes $\mathcal{S}_{\mathrm{sd}} L$ among all the possible variations of $y$, where a variation of $y$ is a smooth curve $s \in(-\epsilon, \epsilon) \longrightarrow y_{s} \in \mathcal{C}_{N,[0, T]}$ with $y_{0}=y$. Denote by

$$
\delta y_{k}(t)=\left.\frac{d y_{s}}{d s}(k, t)\right|_{s=0}
$$

where we use the notation $y_{k}(t)=y(k, t)$. Observe that $y_{s}(0, t)=y_{0}(t)$ and $y_{s}(N, t)=y_{N}(t)$ for all $s$. We will also use the following notation

$$
\begin{aligned}
& D_{i} L_{(k, k+1)}(t)=\left(D_{i} L\right)\left(y_{k}(t), \dot{y}_{k}(t), y_{k+1}(t), \dot{y}_{k+1}(t)\right), \\
& \tilde{D}_{j} L_{(k, k+1)}(t)=\left(\tilde{D}_{j} L\right)\left(y_{k}(t), \dot{y}_{k}(t), y_{k+1}(t), \dot{y}_{k+1}(t)\right)
\end{aligned}
$$

for $i \in\{1, \ldots, 4\}$ and $j \in\{1,2\}$.

Extremizing the semi-discrete action function among all the possible variations, we find that

$$
\begin{aligned}
& \left.\frac{d}{d s}\right|_{s=0}\left(\mathcal{S}_{\mathrm{sd}} L\left(y_{s}\right)\right)=\left.\frac{d}{d s}\right|_{s=0} \int_{0}^{T}\left[\sum_{k=0}^{N-1} L\left(y_{s}(k, t), \dot{y}_{s}(k, t), y_{s}(k+1, t), \dot{y}_{s}(k+1, t)\right)\right] d t \\
& =\int_{0}^{T} \sum_{k=1}^{N-1}\left[D_{3} L_{(k-1, k)}(t) \delta y_{k}(t)+D_{4} L_{(k-1, k)}(t) \frac{d}{d t} \delta y_{k}(t)\right. \\
& \left.+D_{1} L_{(k, k+1)}(t) \delta y_{k}(t)+D_{2} L_{(k, k+1)}(t) \frac{d}{d t} \delta y_{k}(t)\right] d t \\
& =\int_{0}^{T}\left[\sum_{k=1}^{N-1}\left(D_{3} L_{(k-1, k)}(t)+D_{1} L_{(k, k+1)}(t)-\frac{d}{d t}\left(D_{4} L_{(k-1, k)}(t)+D_{2} L_{(k, k+1)}(t)\right)\right) \delta y_{k}(t)\right] d t \\
& +\left.\sum_{k=1}^{N-1}\left(D_{4} L_{(k-1, k)}(t)+D_{2} L_{(k, k+1)}(t)\right) \delta y_{k}(t)\right|_{0} ^{T} .
\end{aligned}
$$

Therefore, the semi-discrete Euler-Lagrange equations are:

$$
\begin{aligned}
D_{3} L_{(k-1, k)}(t)+D_{1} L_{(k, k+1)}(t)-\frac{d}{d t}\left(D_{4} L_{(k-1, k)}(t)+D_{2} L_{(k, k+1)}(t)\right) & =0, \quad 1 \leq k \leq N-1 \\
D_{4} L_{(k-1, k)}(0)+D_{2} L_{(k, k+1)}(0) & =0 \\
D_{4} L_{(k-1, k)}(T)+D_{2} L_{(k, k+1)}(T) & =0
\end{aligned}
$$

The first equations represent a system of second order differential equations of the form:

$$
F\left(y_{k-1}(t), y_{k}(t), y_{k+1}(t) ; \dot{y}_{k-1}(t), \dot{y}_{k}(t), \dot{y}_{k+1}(t) ; \ddot{y}_{k-1}(t), \ddot{y}_{k}(t), \ddot{y}_{k+1}(t)\right)=0
$$


When the matrix $\left(D_{24} L_{(j-1, j)}(t)\right)$ is regular then we may locally write these equations as

$$
\ddot{y}_{k+1}(t)=G\left(y_{k-1}(t), y_{k}(t), y_{k+1}(t) ; \dot{y}_{k-1}(t), \dot{y}_{k}(t), \dot{y}_{k+1}(t) ; \ddot{y}_{k-1}(t), \ddot{y}_{k}(t)\right)
$$

Then, for enough small $T$, the semi-discrete flow $\Upsilon$ :

$$
\begin{aligned}
& \Upsilon: \quad Q^{[0, T]} \times Q^{[0, T]} \longrightarrow Q^{[0, T]} \times Q^{[0, T]} \\
& \left(y_{k-1}(\cdot), y_{k}(\cdot)\right) \longmapsto\left(y_{k}(\cdot), y_{k+1}(\cdot)\right)
\end{aligned}
$$

is well-defined (since Equations (5.2) appear as a system of explicit differential equations), where $\left(y_{k-1}(\cdot), y_{k}(\cdot), y_{k+1}(\cdot)\right)$ satisfies Equations (5.1) and

$$
Q^{[0, T]}=\left\{z:[0, T] \rightarrow Q / z \in C^{2}([0, T])\right\} .
$$

5.1.1. Symplecticity. Define the Poincaré-Cartan 1-forms $\Theta_{L}^{-}, \Theta_{L}^{+} \in \Lambda^{1}\left(Q^{[0, T]} \times Q^{[0, T]}\right)$ as follows

$$
\begin{aligned}
\Theta_{L}^{-}(X(\cdot), Y(\cdot)) & =-\int_{0}^{T}\left(\tilde{D}_{1} L_{(0,1)}(t) \dot{X}(t)\right) d t \\
& =-\int_{0}^{T}\left(D_{1} L_{(0,1)}(t) X(t)+D_{2} L_{(0,1)}(t) \dot{X}(t)\right) d t \\
& =-\int_{0}^{T}\left(D_{1} L_{(0,1)}(t)-\frac{d}{d t}\left(D_{2} L_{(0,1)}(t)\right)\right) X(t) d t-\left.D_{2} L_{(0,1)}(t) X(t)\right|_{0} ^{T} \\
\Theta_{L}^{+}(X(\cdot), Y(\cdot)) & =\int_{0}^{T}\left(\tilde{D}_{2} L_{(0,1)}(t) \dot{Y}(t)\right) d t \\
& =\int_{0}^{T}\left(D_{3} L_{(0,1)}(t) Y(t)+D_{4} L_{(0,1)}(t) \dot{Y}(t)\right) d t \\
& =\int_{0}^{T}\left(D_{3} L_{(0,1)}(t)-\frac{d}{d t}\left(D_{4} L_{(0,1)}(t)\right)\right) Y(t) d t+\left.D_{4} L_{(0,1)}(t) Y(t)\right|_{0} ^{T}
\end{aligned}
$$

Then, we have

$$
\Theta_{L}^{+}(X(\cdot), Y(\cdot))-\Theta_{L}^{-}(X(\cdot), Y(\cdot))=d\left[\int_{0}^{T} L\left(y_{0}(t), \dot{y}_{0}(t), y_{1}(t), \dot{y}_{1}(t)\right) d t\right](X(\cdot), Y(\cdot))
$$

Therefore, there exists a well-defined 2-form $\Omega_{L}$

$$
\Omega_{L}=d \Theta_{L}^{-}=d \Theta_{L}^{+}
$$

As a consequence of Equations (5.1) we deduce that $\Upsilon^{*} \Theta_{L}^{-}=\Theta_{L}^{+}$and

$$
\Upsilon^{*} \Omega_{L}=\Omega_{L}
$$

5.1.2. Legendre transformations. Define the semi-discrete Legendre transformations as

$$
\begin{aligned}
\operatorname{Leg}_{L}^{-}: Q^{[0, T]} \times Q^{[0, T]} & \longrightarrow T^{*} Q^{[0, T]} \\
\left(y_{0}(\cdot), y_{1}(\cdot)\right) & \longmapsto \operatorname{Leg}_{L}^{-}\left(y_{0}(\cdot), y_{1}(\cdot)\right): T_{y_{0}(\cdot) Q^{[0, T]}} \longrightarrow \mathbb{R} \\
X(\cdot) & \longmapsto \Theta_{L}^{-}(X(\cdot), \mathbf{0})
\end{aligned}
$$

and

$$
\begin{aligned}
\operatorname{Leg}_{L}^{+}: Q^{[0, T]} \times Q^{[0, T]} & \longrightarrow T^{*} Q^{[0, T]} \\
\left(y_{0}(\cdot), y_{1}(\cdot)\right) & \longmapsto \operatorname{Leg}_{L}^{+}\left(y_{0}(\cdot), y_{1}(\cdot)\right): T_{y_{1}(\cdot) Q^{[0, T]}} \longrightarrow \mathbb{R} \\
Y(\cdot) & \longmapsto \Theta_{L}^{+}(\mathbf{0}, Y(\cdot))
\end{aligned}
$$

Denote by $\Theta_{Q}$ and $\Omega_{Q}$ the Liouville 1-form and the canonical symplectic 2-form, respectively, on $T^{*} Q^{[0, T]}$ defined by

$$
\begin{aligned}
\Theta_{Q}(\tilde{\alpha}(\cdot))(\tilde{X}(\cdot)) & =\tilde{\alpha}(\cdot)\left(\left(\tau_{Q^{[0, T]}}\right)_{*}(\tilde{X}(\cdot))\right)=\int_{0}^{T} \tilde{\alpha}\left(\left(\tau_{Q}\right)_{*}(\tilde{X}(t))\right) d t \\
\Omega_{Q} & =d \Theta_{Q}
\end{aligned}
$$

where $\tau_{Q}: T^{*} Q \longrightarrow Q$ and $\tau_{Q^{[0, T]}}: T^{*} Q^{[0, T]} \rightarrow Q^{[0, T]}$ are the canonical projections. Then, it is easy to prove that

$$
\left(\operatorname{Leg}_{L}^{-}\right)^{*} \Theta_{Q}=\Theta_{L}^{-}, \quad\left(\operatorname{Leg}_{L}^{-}\right)^{*} \Theta_{Q}=\Theta_{L}^{+}, \quad\left(\operatorname{Leg}_{L}^{-}\right)^{*} \Omega_{Q}=\Omega_{L}
$$


5.1.3. Momentum mapping. Suppose that the Lagrangian is invariant by a Lie group of symmetries, that is, if $\phi: G \times Q \rightarrow Q$ is the action of a Lie group then

$$
L\left(T_{q} \phi_{g}\left(v_{q}\right), T_{q^{\prime}} \phi_{g}\left(v_{q^{\prime}}\right)\right)=L\left(v_{q}, v_{q^{\prime}}\right), \quad \forall v_{q}, v_{q^{\prime}} \in T Q, \quad q, q^{\prime} \in Q
$$

Infinitesimally, this condition means that

$$
\xi_{T(Q \times Q)}\left(v_{q}, v_{q^{\prime}}\right)(L)=\left(\xi_{T Q}\left(v_{q}\right)+\xi_{T Q}\left(v_{q^{\prime}}\right)\right)(L)=0, \quad \forall \xi \in \mathfrak{g}
$$

where $\xi_{T Q}$ is the infinitesimal generator of the lifted action $\phi^{T}: G \times T Q \rightarrow T Q$. In particular,

$$
\left(\xi_{T Q}\left(y_{k-1}(t), \dot{y}_{k-1}(t)\right)+\xi_{T Q}\left(y_{k}(t), \dot{y}_{k}(t)\right)\right)(L)=0
$$

or, in other words,

$$
\begin{aligned}
& D_{1} L_{(k-1, k)}(t) \xi_{Q}\left(y_{k-1}(t)\right)+D_{2} L_{(k-1, k)}(t) \frac{d}{d t}\left(\xi_{Q}\left(y_{k-1}(t)\right)\right) \\
& +D_{3} L_{(k-1, k)}(t) \xi_{Q}\left(y_{k}(t)\right)+D_{4} L_{(k-1, k)}(t) \frac{d}{d t}\left(\xi_{Q}\left(y_{k}(t)\right)\right)=0
\end{aligned}
$$

where $\xi_{Q}$ is the infinitesimal generator of the action $\phi$ corresponding to $\xi$ (note that $\xi_{T Q}$ is the complete lift of $\left.\xi_{Q}\right)$. Therefore

$$
\begin{aligned}
& D_{3} L_{(k-1, k)}(t) \xi_{Q}\left(y_{k}(t)\right)+D_{1} L_{(k-1, k)}(t) \xi_{Q}\left(y_{k-1}(t)\right) \\
& -\frac{d}{d t}\left(D_{4} L_{(k-1, k)}(t)\right) \xi_{Q}\left(y_{k}(t)\right)-\frac{d}{d t}\left(D_{2} L_{(k-1, k)}(t)\right) \xi_{Q}\left(y_{k-1}(t)\right) \\
& +\frac{d}{d t}\left[D_{4} L_{(k-1, k)}(t) \xi_{Q}\left(y_{k}(t)\right)+D_{2} L_{(k-1, k)}(t) \xi_{Q}\left(y_{k-1}(t)\right)\right]=0
\end{aligned}
$$

Subtracting first expression in (5.1) applied to $\xi_{T(Q \times Q)}$ and the above equation we deduce that

$$
\begin{aligned}
& D_{1} L_{(k, k+1)}(t) \xi_{Q}\left(y_{k}(t)\right)-\frac{d}{d t}\left(D_{2} L_{(k, k+1)}(t)\right) \xi_{Q}\left(y_{k}(t)\right) \\
= & D_{1} L_{(k-1, k)}(t) \xi_{Q}\left(y_{k-1}(t)\right)-\frac{d}{d t}\left(D_{2} L_{(k-1, k)}(t)\right) \xi_{Q}\left(y_{k-1}(t)\right) \\
& +\frac{d}{d t}\left[D_{4} L_{(k-1, k)}(t) \xi_{Q}\left(y_{k}(t)\right)+D_{2} L_{(k-1, k)}(t) \xi_{Q}\left(y_{k-1}(t)\right)\right] .
\end{aligned}
$$

Therefore, integrating and using the two last equations in (5.1) we obtain the following preservation law

$$
\begin{aligned}
& \int_{0}^{T}\left[D_{1} L_{(k-1, k)}(t)-\frac{d}{d t}\left(D_{2} L_{(k-1, k)}(t)\right)\right] \xi_{Q}\left(y_{k-1}(t)\right) d t+\left.D_{2} L_{(k-1, k)}(t) \xi_{Q}\left(y_{k-1}(t)\right)\right|_{0} ^{T} \\
& =\int_{0}^{T}\left[D_{1} L_{(k, k+1)}(t)-\frac{d}{d t}\left(D_{2} L_{(k, k+1)}(t)\right)\right] \xi_{Q}\left(y_{k}(t)\right) d t+\left.D_{2} L_{(k, k+1)}(t) \xi_{Q}\left(y_{k}(t)\right)\right|_{0} ^{T} .
\end{aligned}
$$

Note that this equation may be written as

$$
\Theta_{L}^{-}\left(y_{k-1}(\cdot), y_{k}(\cdot)\right)\left(\xi_{Q} \circ y_{k-1}, \xi_{Q} \circ y_{k}\right)=\Theta_{L}^{-}\left(y_{k}(\cdot), y_{k+1}(\cdot)\right)\left(\xi_{Q} \circ y_{k}, \xi_{Q} \circ y_{k+1}\right) .
$$

5.1.4. The nonlinear wave equation. First point of view. The semi-discrete Euler-Lagrange equations for the Lagrangian:

$$
L_{s d}\left(u_{0}, u_{1},\left(u_{0}\right)_{t},\left(u_{1}\right)_{t}\right)=\frac{1}{2}\left(\frac{\left(u_{0}\right)_{t}+\left(u_{1}\right)_{t}}{2}\right)^{2}-\sigma\left(\frac{u_{1}-u_{0}}{h}\right)-f\left(\frac{u_{1}+u_{0}}{2}\right)
$$

are:

$$
\begin{aligned}
& \frac{\left(u_{k-1}\right)_{t t}+2\left(u_{k}\right)_{t t}+\left(u_{k+1}\right)_{t t}}{4}-\frac{1}{h}\left[\sigma^{\prime}\left(\frac{u_{k+1}-u_{k}}{h}\right)-\sigma^{\prime}\left(\frac{u_{k}-u_{k-1}}{h}\right)\right] \\
& -\frac{1}{2}\left[f^{\prime}\left(\frac{u_{k}+u_{k-1}}{2}\right)+f^{\prime}\left(\frac{u_{k+1}+u_{k}}{2}\right)\right]=0, \quad 1 \leq k \leq N-1
\end{aligned}
$$


with boundary conditions

$$
\begin{aligned}
& u_{0}(t) \text { and } u_{1}(t) \text { fixed } \\
& \left(u_{k-1}\right)_{t}(0)+2\left(u_{k}\right)_{t}(0)+\left(u_{k+1}\right)_{t}(0)=0 \\
& \left(u_{k-1}\right)_{t}(T)+2\left(u_{k}\right)_{t}(T)+\left(u_{k+1}\right)_{t}(T)=0
\end{aligned}
$$

5.2. Variational calculus on $\widetilde{\mathcal{C}}_{N,[0, T]}$. Instead of the set of functions $\mathcal{C}_{N,[0, T]}$ we consider the set $\widetilde{\mathcal{C}}_{N,[0, T]}$ where now the values at time 0 and $T$ are fixed. As in the previous case, we have the semi-discrete action $\widetilde{\mathcal{S}}_{\mathrm{Sd}} L: \widetilde{\mathcal{C}}_{N,[0, T]} \longrightarrow \mathbb{R}:$

$$
\widetilde{\mathcal{S}}_{\mathrm{Sd}} L(y(\cdot, \cdot))=\int_{0}^{T}\left[\sum_{k=0}^{N-1} L(y(k, t), \dot{y}(k, t), y(k+1, t), \dot{y}(k+1, t))\right] d t .
$$

Definition 5.2. A function $y \in \widetilde{\mathcal{C}}_{N,[0, T]}$ is a solution of the semi-discrete variational problem determined by $L$ if and only if it is a critical point of the Lagrangian system defined by $\widetilde{\mathcal{S}}_{s d} L$.

Therefore

$$
\begin{aligned}
& \left.\frac{d}{d s}\right|_{s=0}\left(\widetilde{\mathcal{S}}_{\mathrm{Sd}} L\left(y_{s}\right)\right)=\left.\frac{d}{d s}\right|_{s=0} \int_{0}^{T}\left[\sum_{k=0}^{N-1} L\left(y_{s}(k, t), \dot{y}_{s}(k, t), y_{s}(k+1, t), \dot{y}_{s}(k+1, t)\right)\right] d t \\
& =\int_{0}^{T} \sum_{k=1}^{N-1}\left[D_{3} L_{(k-1, k)}(t) \delta y_{k}(t)+D_{4} L_{(k-1, k)}(t) \frac{d}{d t} \delta y_{k}(t)\right. \\
& \left.+D_{1} L_{(k, k+1)}(t) \delta y_{k}(t)+D_{2} L_{(k, k+1)}(t) \frac{d}{d t} \delta y_{k}(t)\right] d t \\
& +\int_{0}^{T}\left[D_{1} L_{(0,1)}(t) \delta y_{0}(t)+D_{2} L_{(0,1)}(t) \frac{d}{d t} \delta y_{0}(t)\right] d t \\
& +\int_{0}^{T}\left[D_{3} L_{(N-1, N)}(t) \delta y_{N}(t)+D_{4} L_{(N-1, N)}(t) \frac{d}{d t} \delta y_{N}(t)\right] d t \\
& =\int_{0}^{T}\left[\sum_{k=1}^{N-1}\left(D_{3} L_{(k-1, k)}(t)+D_{1} L_{(k, k+1)}(t)-\frac{d}{d t}\left(D_{4} L_{(k-1, k)}(t)+D_{2} L_{(k, k+1)}(t)\right)\right) \delta y_{k}(t)\right] d t \\
& +\int_{0}^{T}\left[D_{1} L_{(0,1)}(t)-\frac{d}{d t}\left(D_{2} L_{(0,1)}(t)\right)\right] \delta y_{0}(t) d t \\
& +\int_{0}^{T}\left[D_{3} L_{(N-1, N)}(t)-\frac{d}{d t}\left(D_{4} L_{(N-1, N)}(t)\right)\right] \delta y_{N}(t) d t .
\end{aligned}
$$

Therefore, the semi-discrete Euler-Lagrange equations are:

$$
\begin{aligned}
D_{3} L_{(k-1, k)}(t)+D_{1} L_{(k, k+1)}(t)-\frac{d}{d t}\left(D_{4} L_{(k-1, k)}(t)+D_{2} L_{(k, k+1)}(t)\right) & =0, \quad 1 \leq k \leq N-1 \\
D_{1} L_{(0,1)}(t)-\frac{d}{d t}\left(D_{2} L_{(0,1)}(t)\right) & =0 \\
D_{3} L_{(N-1, N)}(t)-\frac{d}{d t}\left(D_{4} L_{(N-1, N)}(t)\right) & =0
\end{aligned}
$$

These are precisely the Euler-Lagrange equations

$$
\frac{\partial \widetilde{L}}{\partial y_{k}}-\frac{d}{d t}\left(\frac{\partial \widetilde{L}}{\partial \dot{y}_{k}}\right)=0, \quad 0 \leq k \leq N
$$

for the Lagrangian $\widetilde{L}:(T Q)^{N+1} \simeq T Q^{N+1} \longrightarrow \mathbb{R}$ defined by

$$
\widetilde{L}\left(y_{0}, \ldots, y_{N},\left(y_{0}\right)_{t}, \ldots,\left(y_{N}\right)_{t}\right)=\sum_{k=0}^{N-1} L\left(y_{k},\left(y_{k}\right)_{t}, y_{k+1},\left(y_{k+1}\right)_{t}\right),
$$


where $(T Q)^{N+1}$ stands for the Cartesian Product of $N+1$ copies of $T Q$.

We define

- The Poincaré Cartan 1-form $\Theta_{\tilde{L}} \in \Lambda^{1}\left(T Q^{N+1}\right)$ :

$$
\Theta_{\tilde{L}}=D_{2} L_{(0,1)} d y_{0}+\sum_{k=1}^{N-1}\left[D_{4} L_{(k-1, k)}+D_{2} L_{(k, k+1)}\right] d y_{k}+D_{4} L_{(N-1, N)} d y_{N}
$$

- The Poincaré-Cartan 2-form $\Omega_{\tilde{L}}=d \Theta_{\tilde{L}}$.

- The energy function

$$
E_{\tilde{L}}=D_{2} L_{(0,1)}\left(y_{0}\right)_{t}+\sum_{k=1}^{N-1}\left[D_{4} L_{(k-1, k)}+D_{2} L_{(k, k+1)}\right]\left(y_{k}\right)_{t}+D_{4} L_{(N-1, N)}\left(y_{N}\right)_{t}-\tilde{L}
$$

We say that the system is regular if and only if the 2 -form $\Omega_{\tilde{L}}$ is a symplectic 2 -form. Locally the regularity is equivalent to the non-singularity of the Hessian matrix $\left(\frac{\partial^{2} \tilde{L}}{\partial\left(y_{k}\right)_{t} \partial\left(y_{l}\right)_{t}}\right) 0 \leq k, l \leq N$. In such a case, there exists a unique vector field $\xi_{\tilde{L}} \in \mathfrak{X}\left(T(Q)^{N+1}\right)$ such that

$$
i_{\xi_{\tilde{L}}} \Omega_{\tilde{L}}=d E_{\tilde{L}}
$$

Moreover, the integral curves of $\xi_{\tilde{L}}$ are the tangent lifts of the solutions of the semi-discrete Euler Lagrange equations (5.5).

In many situations, the 2 -form $\Omega_{\tilde{L}}$ is not symplectic. Then, (5.6) has no solution, in general, and even if it exists it will not be unique. Let $b_{\tilde{L}}: T Q^{N+1} \longrightarrow T^{*} Q^{N+1}$ be the map defined by $b_{\tilde{L}}(X)=i_{X} \Omega_{\tilde{L}}$. It may happen that $\Omega_{\tilde{L}}$ is not surjective. We denote by $\operatorname{ker} \Omega_{\tilde{L}}$ the kernel of $b_{\tilde{L}}$, i.e., $\operatorname{ker} b_{\tilde{L}}=\operatorname{ker} \Omega_{\tilde{L}}$.

In [4, 5, Gotay and Nester have developed a constraint algorithm for presymplectic systems which is an intrinsic version of the classical Dirac-Bergmann algorithm . They consider the set of points $P_{2}$ of $P_{1}=T Q^{N+1}$ where (5.6) has a solution and suppose that this set $P_{2}$ is a submanifold of $P_{1}$. Nevertheless, these solutions on $P_{2}$ may not be tangent to $P_{2}$. Then, we have to restrict $P_{2}$ to a submanifold where the solutions of (5.6) are tangent to $P_{2}$. Proceeding further we obtain a sequence of submanifolds:

$$
\cdots \rightarrow P_{k} \rightarrow \cdots \rightarrow P_{2} \rightarrow P_{1}=T Q^{N+1} .
$$

Alternatively, these constraint submanifolds may be described as follows:

$$
P_{i}=\left\{p \in P_{i-1} / d E_{\tilde{L}}(p)(v)=0, \forall v \in T_{p} P_{i-1}^{\perp}\right\},
$$

where

$$
T_{p} P_{i-1}^{\perp}=\left\{v \in T_{p} P_{1} / \Omega_{\tilde{L}}(x)(u, v)=0, \forall u \in T_{p} P_{i-1}\right\} .
$$

We call $P_{2}$ the secondary constraint submanifold, $P_{3}$ the tertiary constraint submanifold, and, in general, $P_{i}$ is the $i$-ary constraint submanifold.

If the algorithm stabilizes, i.e. there exists a positive integer $k \in \mathbb{N}$ such that $P_{k}=P_{k+1}$ and $\operatorname{dim} P_{k} \neq 0$, then we have a final constraint submanifold $P_{f}=P_{k}$, on which exists a vector field $X$ such that

$$
\left(i_{X} \Omega_{\tilde{L}}=d E_{\tilde{L}}\right)_{/ P_{f}} .
$$

If $\xi$ is a solution of (5.7) then every arbitrary solution on $P_{f}$ is of the form $\xi^{\prime}=\xi+Y$, where $Y \in\left(\operatorname{ker} \Omega_{\tilde{L}} \cap T P_{f}\right)$.

Another interesting aspect of this theory is that, in any case, regular or singular, since the Lagrangian $\tilde{L}$ is autonomous, then $\xi\left(E_{\tilde{L}}\right)=0$ for any solution of Equation (5.7). In particular, for $\xi_{\tilde{L}}$, we have that

$$
\frac{d}{d t}\left[D_{2} L_{(0,1)} \dot{y}_{0}+\sum_{k=1}^{N-1}\left[D_{4} L_{(k-1, k)}+D_{2} L_{(k, k+1)}\right] \dot{y}_{k}+D_{4} L_{(N-1, N)} \dot{y}_{N}-\tilde{L}\right]=0
$$


and the energy $E_{\tilde{L}}$ is a constant of the motion.

5.2.1. Legendre transformation. Define the semi-discrete Legendre transformation as

$\mathbb{F} \tilde{L}:$ $T Q^{N+1} \longrightarrow T^{*} Q^{N+1}$

$\left(y_{0}, \ldots, y_{N},\left(y_{0}\right)_{t}, \ldots,\left(y_{N}\right)_{t}\right) \longmapsto\left(y_{0}, \ldots, y_{N} ; p_{0}, \ldots, p_{N}\right)$

where

$$
p_{0}=D_{2} L_{(0,1)}, \ldots, p_{k}=D_{4} L_{(k-1, k)}+D_{2} L_{(k, k+1)}, \ldots, p_{N}=D_{4} L_{(N-1, N)}
$$

If $\lambda_{Q^{N+1}}$ and $\omega_{Q^{N+1}}$ are the Liouville 1 -form and the canonical symplectic 2 -form on $T^{*} Q^{N+1}$ then

$$
\mathbb{F} \tilde{L}^{*} \lambda_{Q^{N+1}}=\Theta_{\widetilde{L}}, \quad \mathbb{F} \tilde{L}^{*} \omega_{Q^{N+1}}=\Omega_{\widetilde{L}}
$$

Moreover, if the Legendre transformation $\mathbb{F} \tilde{L}$ is a global diffeomorphism, we will say that the Lagrangian $\tilde{L}$ is hyperregular. In this case it is well defined the function $H=E_{\tilde{L}} \circ(\mathbb{F} \tilde{L})^{-1}$.

Therefore, we have a Hamiltonian representation of the equations of motion (5.5):

$$
i_{X_{H}} \omega_{Q^{N+1}}=d H
$$

or, in coordinates,

$$
\dot{y}_{i}=\frac{\partial H}{\partial p_{i}}, \quad \dot{p}_{i}=-\frac{\partial H}{\partial y^{i}}, \quad 0 \leq i \leq N
$$

Obviously, $\xi_{\tilde{L}}$ and $X_{H}$ are $\mathbb{F} \tilde{L}$-related, i.e. $(\mathbb{F} \tilde{L})_{*} \xi_{\tilde{L}}=X_{H}$.

If the lagrangian $\tilde{L}$ is singular, $\mathbb{F} \tilde{L}$ is not a diffeomorphism. However, we may assume that $\tilde{L}$ is almost regular, i.e., $M_{1}=\mathbb{F} \tilde{L}\left(T Q^{N+1}\right)$ is a submanifold of $T^{*} Q^{N+1}$ and, $\mathbb{F} \tilde{L}$ is a submersion onto $M_{1}$ with connected fibers. The submanifold $M_{1}$ will be called the primary constraint submanifold.

Since the Lagrangian is almost regular, the energy $E_{\tilde{L}}$ is constant along the fibers of $\mathbb{F} \tilde{L}$. Therefore, $E_{\tilde{L}}$ projects onto a function $H_{M_{1}}$ on $M_{1}$ :

$$
H_{M_{1}}(\mathbb{F} \tilde{L}(p))=E_{\tilde{L}}(p), \forall p \in T Q^{N+1} .
$$

If we denote by $i_{M_{1}}: M_{1} \longrightarrow T^{*} Q^{N+1}$ the embedding of $M_{1}$ into $T^{*} Q^{N+1}$, then we obtain a presymplectic system $\left(M_{1},\left(i_{M_{1}}\right)^{*} \omega_{Q^{N+1}}, d H_{M_{1}}\right)$. If we now apply the constraint algorithm to it, we shall obtain the following sequence of constraint submanifolds:

$$
\cdots \rightarrow M_{k} \rightarrow \cdots \rightarrow M_{2} \rightarrow M_{1}
$$

as in the Lagrangian side. Denote by $M_{f}$ the final constraint submanifold (if it exists) for this presymplectic system. In $M_{f}$ there exists at least a vector field $X \in \mathfrak{X}\left(M_{f}\right)$ such that

$$
\left(i_{X}\left[\left(i_{M_{1}}\right)^{*} \omega_{Q^{N+1}}\right]=d H_{M_{1}}\right)_{/ M_{f}}
$$

The Legendre map restricts to each submanifold $P_{i}, i \geq 1$, of $T Q^{N+1}$ and then we obtain a family of surjective submersions $(\mathbb{F} \tilde{L})_{i}: P_{i} \longrightarrow M_{i}$ which relates the constraint submanifolds $P_{i}$ and $M_{i}$, in particular, $P_{f}$ and $M_{f}$.

5.2.2. Momentum preservation. Suppose, as in Subsection 5.1.3 that the Lagrangian $L$ is invariant by a Lie group of symmetries. Infinitesimally, this conditions implies that

$$
\xi_{T Q^{N+1}}\left(v_{q_{0}}, \ldots, v_{q_{N}}\right)(\widetilde{L})=0, \quad \forall \xi \in \mathfrak{g}
$$

In such a case, applying the classical Noether theorem, we deduce that the function $F: T Q^{N+1} \longrightarrow$ $\mathbb{R}$ defined by

$$
F=\Theta_{\tilde{L}}\left(\xi_{T Q^{N+1}}\right)
$$

is a constant of the motion. Explicitly,

$$
\begin{aligned}
& F\left(y_{0}, \dot{y}_{0}, \ldots, y_{N}, \dot{y}_{N}\right)=D_{2} L_{(0,1)} \xi_{Q}\left(y_{0}\right) \\
& +\sum_{k=1}^{N-1}\left[D_{4} L_{(k-1, k)}+D_{2} L_{(k, k+1)}\right] \xi_{Q}\left(y_{k}\right)+D_{4} L_{(N-1, N)} \xi_{Q}\left(y_{N}\right)
\end{aligned}
$$


5.2.3. The nonlinear wave equation. Second point of view. Consider the semidiscrete Lagrangian

$$
L_{s d}\left(u_{0}, u_{1},\left(u_{0}\right)_{t},\left(u_{1}\right)_{t}\right)=\frac{1}{2}\left(\frac{\left(u_{0}\right)_{t}+\left(u_{1}\right)_{t}}{2}\right)^{2}-\sigma\left(\frac{u_{1}-u_{0}}{h}\right)-f\left(\frac{u_{1}+u_{0}}{2}\right)
$$

for the nonlinear wave equation. Then the Euler-Lagrange equations are:

$$
\frac{1}{4}\left(\left(u_{0}\right)_{t t}+\left(u_{1}\right)_{t t}\right)-\frac{1}{h}\left[\sigma^{\prime}\left(\frac{u_{1}-u_{0}}{h}\right)\right]+\frac{1}{2} f^{\prime}\left(\frac{u_{0}+u_{1}}{2}\right)=0
$$

$$
\begin{aligned}
& \frac{\left(u_{k-1}\right)_{t t}+2\left(u_{k}\right)_{t t}+\left(u_{k+1}\right)_{t t}}{4}-\frac{1}{h}\left[\sigma^{\prime}\left(\frac{u_{k+1}-u_{k}}{h}\right)-\sigma^{\prime}\left(\frac{u_{k}-u_{k-1}}{h}\right)\right] \\
& +\frac{1}{2}\left[f^{\prime}\left(\frac{u_{k}+u_{k-1}}{2}\right)+f^{\prime}\left(\frac{u_{k+1}+u_{k}}{2}\right)\right]=0, \quad 1 \leq k \leq N-1
\end{aligned}
$$

$$
\frac{1}{4}\left(\left(u_{N}\right)_{t t}+\left(u_{N-1}\right)_{t t}\right)+\frac{1}{h}\left[\sigma^{\prime}\left(\frac{u_{N}-u_{N-1}}{h}\right)\right]+\frac{1}{2} f^{\prime}\left(\frac{u_{N-1}+u_{N}}{2}\right)=0 .
$$

The Lagrangian $\tilde{L}$ is

$$
\begin{aligned}
\tilde{L}\left(u_{0}, \ldots, u_{N} ;\left(u_{0}\right)_{t}, \ldots,\left(u_{N}\right)_{t}\right)= & \sum_{k=1}^{N}\left[\frac{1}{2}\left(\frac{\left(u_{k-1}\right)_{t}+\left(u_{k}\right)_{t}}{2}\right)^{2}\right. \\
& \left.-\sigma\left(\frac{u_{k}-u_{k-1}}{h}\right)-f\left(\frac{u_{k}+u_{k-1}}{2}\right)\right]
\end{aligned}
$$

and the Poincaré-Cartan 1-form is

$$
\begin{aligned}
\Theta_{\tilde{L}}= & \frac{1}{4}\left[\left(\left(u_{0}\right)_{t}+\left(u_{1}\right)_{t}\right) d u_{0}+\sum_{k=1}^{N-1}\left(\left(u_{k-1}\right)_{t}+2\left(u_{k}\right)_{t}+\left(u_{k+1}\right)_{t}\right) d u_{k}\right. \\
& \left.+\left(\left(u_{N-1}\right)_{t}+\left(u_{N}\right)_{t}\right) d u_{N}\right]
\end{aligned}
$$

In this case the Poincaré Cartan 2-form $\Omega_{\tilde{L}}=-d \Theta_{\tilde{L}}$ is degenerate with

$$
\operatorname{ker} \Omega_{\tilde{L}}=\operatorname{span}\left\{\sum_{k=0}^{N}(-1)^{k} \frac{\partial}{\partial u_{k}}, \sum_{k=0}^{N}(-1)^{k} \frac{\partial}{\partial\left(u_{k}\right)_{t}}\right\}
$$

and, therefore, it is necessary to implement the constraint algorithm. We will study in a future paper the constraint algorithm for this and other examples.

\section{Conclusions And outlook}

In this paper we have elucidated the geometrical framework for semi-discrete Mechanics, an useful tool for numerical simulation of Classical Field theories. For simplicity, we only have studied the case of the lagrangian $L_{s d}: T Q \times T Q \simeq T(Q \times Q) \longrightarrow \mathbb{R}$ but also it is possible to consider extensions of this theory to the case of Lagrangians $L_{s d}: \mathcal{P}^{\tau} G \longrightarrow \mathbb{R}$ where $\mathcal{P}^{\tau} G$ is the prolongation of a Lie groupoid $G \rightrightarrows M$ over the fibration $\tau: A G \longrightarrow \mathbb{R}, A G$ being the Lie algebroid associated to the Lie groupoid $G$ (see [9] for more details). This extension may be useful for generating numerical schemes for Field Theories modeled on Lie algebroids (see [14]).

Moreover, the semi-discrete lagrangian $L_{s d}: T Q \times T Q \longrightarrow \mathbb{R}$ is adapted for field theories whose lagrangian $L: Z \longrightarrow \mathbb{R}$ does not depend on the base variables (the base space is assumed bi-dimensional), that is, $L=L\left(y^{i}, z_{\mu}^{i}\right)$. Suppose, for simplicity, that the parameter space is $X=\mathbb{R} \times P$, with $P=\mathbb{R}^{2}$ and $Y=X \times \mathbb{R}$, and the continuous Lagrangian is of the form

$$
L=L\left(t, x^{1}, x^{2}, y, z_{t}, z_{x_{1}}, z_{x_{2}}\right)
$$


Introduce the following natural semi-discretization of $L$

$$
\begin{aligned}
& \left(L_{s d}\right)_{\left(k_{1}, k_{2}\right)}\left(t, y_{\left(k_{1}, k_{2}\right)}(t), y_{\left(k_{1}+1, k_{2}\right)}(t), y_{\left(k_{1}, k_{2}+1\right)}(t) ; \dot{y}_{\left(k_{1}, k_{2}\right)}(t), \dot{y}_{\left(k_{1}+1, k_{2}\right)}(t), \dot{y}_{\left(k_{1}, k_{2}+1\right)}(t)\right) \\
& =L\left(t, k_{1} h_{1}, k_{2} h_{2}, \frac{y_{\left(k_{1}, k_{2}\right)}(t)+y_{\left(k_{1}+1, k_{2}\right)}(t)+y_{\left(k_{1}, k_{2}+1\right)}(t)}{3},\right. \\
& \left.\frac{\dot{y}_{\left(k_{1}, k_{2}\right)}(t)+\dot{y}_{\left(k_{1}+1, k_{2}\right)}(t)+\dot{y}_{\left(k_{1}, k_{2}+1\right)}(t)}{3}, \frac{y_{\left(k_{1}+1, k_{2}\right)}(t)-y_{\left(k_{1}, k_{2}\right)}(t)}{h_{1}}, \frac{y_{\left(k_{1}, k_{2}+1\right)}(t)-y_{\left(k_{1}, k_{2}\right)}(t)}{h_{2}}\right)
\end{aligned}
$$

based on a triangularization of the space $\mathbb{Z} \times \mathbb{Z}$. Then we need to extremize a functional of the type

$$
\begin{array}{r}
\mathcal{S}_{\mathrm{Sd}} L=\int_{0}^{T}\left[\sum _ { ( k _ { 1 } , k _ { 2 } ) \in \{ 0 , \ldots , N - 1 \} \times \{ 0 , \ldots , N - 1 \} } ( L _ { s d } ) _ { ( k _ { 1 } , k _ { 2 } ) } \left(t, y_{\left(k_{1}, k_{2}\right)}(t), y_{\left(k_{1}+1, k_{2}\right)}(t), y_{\left(k_{1}, k_{2}+1\right)}(t) ;\right.\right. \\
\left.\left.\dot{y}_{\left(k_{1}, k_{2}\right)}(t), \dot{y}_{\left(k_{1}+1, k_{2}\right)}(t), \dot{y}_{\left(k_{1}, k_{2}+1\right)}(t)\right)\right] d t
\end{array}
$$

Observe that $\left(L_{s d}\right)_{\left(k_{1}, k_{2}\right)} \in C^{2}\left(\mathbb{R} \times T \mathbb{R}^{3}\right)$ and then new tools must be used as, for instance, cosymplectic geometry and suitable adaptations of the results about Discrete Field Theories (see, for instance, [13, 18]). Also extensions for the case of a non-uniform grid may be considered and in a future paper will be discussed. The generalization of this theory to the case of constrained multisymplectic field theories, as for instance in the case of incompressibility constraints in fluids [11, may be obtained using and adaptation of constrained discrete variational calculus (see [1], for instance).

\section{REFERENCES}

[1] Benito R, Martín de Diego D, Discrete vakonomic Mechanics, Journal of Mathematical Physics, 46 (2005), 083521-083538.

[2] Gotay MJ, Isenberg J, Marsden Je, Momentun maps and classical relativistic fields, Part I: Covariant field theory, MSRI preprint (1997).

[3] Gotay MJ, Isenberg J, Marsden JE, Momentun maps and classical relativistic fields, Part II: Canonical analysis of field theories, MSRI preprint (1999).

[4] Gotay MJ, Nester J, Presymplectic Lagrangian systems I: the constraint algorithm and the equivalence theorem, Ann. Inst. Henri Poincaré 30 (1978), 129-142.

[5] Gotay MJ, Nester J, Presymplectic Lagrangian systems II: the second order differential equation problem, Ann. Inst. Henri Poincaré A 32 (1980), 1-13.

[6] Hairer E, Lubich C, Wanner G, Geometric Numerical Integration, Structure-Preserving Algorithms for Ordinary Differential Equations, Springer Series in Computational Mathematics 31, Springer-Verlag Berlin Heidelberg.

[7] Jaroszkiewicz G, Norton K, Principes of discrete time mechanics II: Classical field theory, J. Phys. A 30 (1997), 3145-3163.

[8] Leimkuhler B, Reich S, Simulating Hamiltonian Dynamics, Cambridge Monographs on Applied and Computational Mathematics, Cambridge University Press 2004.

[9] Marrero JC, Martín de Diego D, Martínez E, Discrete Lagrangian and Hamiltonian Mechanics on Lie groupoids, Nonlinearity 19 (2006), 1313-1348 and Corrigendum 19 (2006), 3003-3004.

[10] Marsden JE, Patrick GW, Shkoller S, Multisymplectic Geometry, variational ntegrators, and Nonlinear PDEs, Commun. Math. Phys. 199 (1996), 351-395.

[11] Marsden Je, Pekarsky S, Shkoller S, West M, Variational Methods, Multisymplectic Geometry and Continuum Mechanics, J. Geometry and Physics 38 (2001) 253-284.

[12] Marsden Je, West M, Discrete Mechanics and variational integrators, Acta Numerica 10 (2001), 357-514.

[13] Mardden JE, Patrick GW, Shkoller S, Multisymplectic Geometry, Variational Integrators, and Nonlinear PDEs, Commun. Math. Phys. 199 (1998), 351-395.

[14] Martínez E, Classical Field Theory on Lie Algebroids: Variational Aspects, J. Phys. A: Math. Gen. 38 (2005), 7145-7160.

[15] Moser J, Veselov AP, Discrete versions of some classical integrable systems and factorization of matrix polynomials, Comm. Math. Phys. 139, (1991), 217-243.

[16] Oliver M, West M, WulfF C, Approximate momentum conservation for spatial semidiscretizations of semilinear wave equations, Numer. Math. 97 (2004) 493-535.

[17] Sanz-Serna JM, Calvo MP, Numerical Hamiltonian Problems, Chapman\& Hall, London 1994.

[18] Vankerschaver J, Cantrijn F, Discrete Lagrangian field theories on Lie groupoids, Preprint math-ph/0511080

[19] Veselov AP, Integrable discrete-time systems and difference operators, Funct. Anal. Appl. 22 (1988), 83-93. 
M. de León: Instituto de Matemáticas y Física Fundamental, Consejo Superior de Investigaciones Científicas, Serrano 123, 28006 Madrid, Spain

E-mail address: mdeleon@imaff.cfmac.csic.es

Juan C. Marrero: Departamento de Matemática Fundamental, Facultad de Matemáticas, UniverSidad de la Laguna, La Laguna, Tenerife, Canary Islands, Spain

E-mail address: jcmarrer@ull.es

D. Martín de Diego: Instituto de Matemáticas y Física Fundamental, Consejo Superior de InvestiGaciones Científicas, Serrano 123, 28006 Madrid, Spain

E-mail address: d.martin@imaff.cfmac.csic.es 